\title{
When to see a doctor for common health problems: distribution patterns of functional health literacy across migrant populations in Switzerland
}

\author{
Sabine Ackermann Rau $\cdot$ Sibel Sakarya $\cdot$ \\ Thomas Abel
}

Received: 26 January 2013/Revised: 19 June 2014 / Accepted: 26 June 2014/ Published online: 11 July 2014

(C) Swiss School of Public Health 2014

\begin{abstract}
Background Knowing when to seek professional help for health problems is considered an important aspect of health literacy. However, little is known about the distribution of help-seeking knowledge in the general population or specific subpopulations.

Methods We analysed data from the "Health Monitoring of the Swiss Migrant Population 2010" and used a short survey tool to study the distribution of help-seeking knowledge. We sampled members of four migrant groups (from Portugal, Turkey, Serbia and Kosovo; $n=2,614$ ). Our tool contained 12 items that addressed common physical and psychological health problems. A total sum score measured help-seeking knowledge. Two sub-scores analysed knowledge related to potential overuse (minor symptoms) or potential underuse (major symptoms). We applied linear regression to show variations in help-seeking knowledge by age, sex, region of origin and length of stay. Results Controlling for self-rated health, we found that region of origin, higher education, female gender and younger age were significantly associated with higher knowledge scores.

Conclusions We present empirical evidence of unequal distribution of help-seeking knowledge across four migrant populations in Switzerland. Our findings contribute to current conceptual developments in health literacy, and provide starting points for future research.
\end{abstract}

S. Ackermann Rau $\cdot$ T. Abel $(\bowtie)$

Institute of Social and Preventive Medicine, University Bern,

Bern, Switzerland

e-mail: abel@ispm.unibe.ch

S. Sakarya

School of Medicine, Department of Public Health, Marmara

University, Istanbul, Turkey
Keywords Health literacy $\cdot$ Help-seeking knowledge . Migrants' health · Switzerland

\section{Introduction}

Knowing when and where to seek professional help for health problems is considered a major component of health literacy (Kickbusch 2001; Nutbeam 2000; Ratzan and Parker 2000). Taking a theoretical perspective, Schulz and Nakamoto refer to this component as "procedural knowledge": the know-how needed to apply factual knowledge and use health information in a specific context (Schulz and Nakamoto 2005). According to Nutbeam's concept of functional, interactive and critical health literacy (Nutbeam 2000), help-seeking knowledge is a component of functional health literacy, relevant in the fields of public health and clinical settings (Nutbeam 2008).

Help-seeking knowledge and health literacy

In a recent study that focused on the patient perspective, Jordan et al. found that knowledge related to help-seeking was perceived as a key component for successfully dealing with health problems (Jordan et al. 2010). Under the theme of "seeking medical help", their study identified factors that affected people's use of medical services, active engagement in doctor-patient exchange, and access to services and information at the appropriate time. Patients described "knowing when to seek health information" as one of seven key abilities crucial to seeking, understanding and using information in the healthcare setting.

Jordan et al. provide a conceptual model of health literacy that enabled us to situate 'knowledge on when and where to seek professional help for health problems' within 
the overall management of problems. Problem management starts with identifying a health issue and leads to its successful resolution. Jordan et al. also described a set of general factors that affect individual knowledge about when and where to seek health information. These include healthcare system factors, socioeconomic factors, patient attitudes and experiences, social support, education and cultural influences. Of those factors, we chose to address education and cultural background in this study.

\section{Health literacy and migration background}

Health literacy models have improved our conceptual understanding of knowledge about when and where to seek help. However, little empirical data exists on the distribution of help-seeking knowledge, or on the factors that influence it on the population level. Data are rare for all population groups in Switzerland, and especially for those with migration background.

The few published studies that have assessed health literacy in migration groups found they had, on average, lower health literacy scores than did native populations (Smith et al. 2012). Those studies dealt mainly with aspects of functional health literacy, and most showed that immigrants had less ability to understand written or oral health information in the language of the country of immigration (Poureslami et al. 2011; Todd and Hoffman-Goetz 2011). Some studies comparing native and migrant populations found a link between lower health literacy and lower selfrated health among immigrants (Berkman et al. 2011; Ng et al. 2011; Omariba and Ng 2011). But these studies really analysed the effect of language proficiency on immigrant health. Omariba and $\mathrm{Ng}$ concluded that health literacy improved the health of both immigrants and non-immigrants, but that language discordance (as a proxy for nonproficiency in the official language) might limit the ability of immigrants to become health literate.

Omariba and Ng's analysis did not address forms of health literacy that may depend less on language proficiency. Some forms of basic factual knowledge (for example, long-term health effects of certain unhealthy behaviours like smoking, or heavy drinking) could increase an individuals' health literacy, but are not strongly dependent on language proficiency. Certain forms of body perception and health self-management might also be more determined by culture-specific systems of values and norms than by language proficiency. These less language-dependent factors may influence the decisions of immigrants to seek professional help for their health problems (Cha et al. 2012; Cooper et al. 2012; Dunn-Navarra et al. 2012; Shaw et al. 2009; Tieu et al. 2010).

Research on health literacy in migrant populations is still scarce. In particular, we lack data on the distribution of different forms of health literacy in migrant populations.
Without data on the distribution of migrants' knowledge about when to seek professional help for health problems, we do not have the evidence to determine if specific health literacy interventions are necessary. In the present paper, we assessed functional health literacy in the form of 'knowing when to seek professional help for common health problems', and report on the distribution of health-seeking knowledge across four migrant groups in Switzerland.

\section{Methods}

\section{Participants}

The survey, "Health Monitoring of the Swiss Migrant Population 2010", was chaired and funded by the Swiss Federal Office of Public Health and the Swiss Federal Office of Migration. It surveyed migrants on their health situation, health behaviour and use of healthcare services (Guggisberg et al. 2011) and included migrants from four European regions, and asylum seekers from one Asian, and one African region. Swiss native persons were not monitored.

Data were drawn from the central migration information system (ZEMIS) where all immigrants in Switzerland were registered. In total, the ZEMIS register delivered 55'706 addresses.

For our analysis, we selected the subsamples of immigrants from the European regions $(n=2,614)$ : Portugal $(n=450)$, Turkey $(n=851)$, Serbia $(n=462)$ and Kosovo $(n=851)$. Those surveyed were between 17 and 74 years old.

Random sampling was applied within each planned quota cell. A preliminary announcement was mailed to all potential participants. Phone numbers were searched automatically in a first step and manually in a second step. If this procedure returned no telephone number, a reply card was mailed that requested the subject to provide a telephone number. This way, the phone numbers of $37.9 \%$ of Portuguese immigrants, $50.2 \%$ of Kosovar immigrants, $54.3 \%$ of Turkish immigrants, and $57.3 \%$ of Serbian immigrants could be identified. A core sample of the permanent resident population was built by disproportional stratification according to sex, duration of stay and place of birth within the regions of origin (size of each quota cell 450 participants): Portugal $(n=450)$; Turkey $(n=451)$; Serbia $(n=462)$; and, Kosovo $(n=451)$. Two smaller additional samples were stratified only by sex (size of each quota cell 200 participants). The first additional sample consisted of recent immigrants (duration of stay less than 2 years) from Turkey $(n=200)$ and Kosovo $(n=200)$. The second additional sample consisted of recently naturalised persons from Turkey $(n=200)$ and Kosovo $(n=200)$, who acquired Swiss citizenship a maximum of 2 years before the survey. 
We added two samples because we assumed that duration of stay would have a significant impact on norms, values, knowledge and behaviour of immigrant groups. Thus we oversampled recent immigrants and naturalised immigrants (with a long duration of stay). The first additional sample, consisting of recent immigrants from Turkey and Kosovo, was merged in the analyses with the core sample. This subsample became an integral part of the core sample (criterion of discrimination was only duration of stay, which was accounted for in the analyses). In contrast, the second additional sample, consisting of recently naturalised persons from Turkey and Kosovo, stayed separate throughout the analyses (criterion of discrimination was not only duration of stay, but also naturalisation).

\section{Data collection}

"Health Monitoring of the Swiss Migrant Population 2010" was conducted in that year, and administered in computer-assisted telephone interviews (CATI) (M.I.S. Trend SA 2011). The surveys spanned several healthrelated topics and were conducted in the immigrants' language to avoid confounding effects of language proficiency on outcomes. The German version of the questionnaire was professionally translated into Portuguese, Turkish, Albanian, and Serbian. Three pre-tests of the first translation were conducted in each language, and written feedback from the interviewers was shared with the translation office. A second professional translator then compared the translations, taking all feedback into account. If there was disagreement over translation, the translators discussed the problem further and resolved it by consensus.

The response rate was $88.4 \%$ in the core sample, and 88.3 or $91.3 \%$ in additional samples 1 and 2, respectively. The withdrawal rate during an interview was $3.8 \%$ in the core sample, and 1 or $1.8 \%$ in samples 1 and 2 , respectively.

\section{Measures}

Participants were presented with 12 short, specific scenarios that depicted 6 common physical symptoms and 6 psychological symptoms, of different degrees of severity. The physical symptoms were drawn from a list used in an earlier study (Cockerham et al. 1986). An expert group, made up of four general practitioners and one clinical psychologist, discussed and evaluated whether those symptoms applied in the current context. When it comes to social and cultural determinants of help-seeking behaviour, symptoms of mental illness may be more sensitive than physical symptoms (Stoeckle et al. 1963). To cover aspects of mental health, we wanted to add scenarios for psychological symptoms to the list. The same expert group proposed and approved six psychological symptoms that are clinically relevant in general practise. Seven of the 12 symptoms (3 physical, 4 psychological) require professional attention from a physician or psychologist. Five symptoms (three physical, two psychological) do not require consultation. There was full agreement among experts on the ratings for all 12 symptoms.

The scenarios that described psychological symptoms followed those that described physical symptoms because mental health problems may be more sensitive issues for respondents, and they may have less experience reporting mental health items. We more or less randomly mixed symptoms that 'require' or 'do not require' professional consultation, in order to avoid any obvious pattern that could have influenced responses.

Participants were asked: "Do you think someone your age should seek medical or psychological help if he/she experiences one of the following problems." Response categories were "yes", "no", "don't know", and "no answer". All questions about symptoms that we applied in this study were conceptualised and applied as formative items (Edwards and Bagozzi 2000). We used each single item to represent help-seeking knowledge. Used as an additive index, a sum score of the measure assessed 'knowledge about when to seek professional help'. We did not make assumptions about latent constructs or variables. Based on the same rationale, we used two sub-scores to explore differences in the distribution of help-seeking knowledge when either minor or major symptoms were addressed.

In the following list of symptoms, need for consultation is indicated by yes/no answers (in parentheses).

Physical symptoms:

- Occasional heartburn after heavy meals (no)

- Cough lasting 3 weeks or longer (yes)

- A sore throat or a head cold with fever up to $38{ }^{\circ} \mathrm{C}$ for 1 or 2 days (no)

- Diarrhoea for 4 or 5 days (yes)

- Shortage of breath even after light exercise (yes)

- Vomiting repeatedly during 1 day (no)

Psychological symptoms:

- Frequent purging (yes)

- Sadness after a relationship crisis (no)

- Depressed mood for most of the day (yes)

- Loss of interest in the things that used to be enjoyable over a period of several weeks (yes)

- Feeling followed by somebody or something (yes)

- Having occasional nightmares (no)

A total sum score of all correct answers to the 12 scenarios measured an individual's knowledge of when to seek professional help. Two sub-scores assessed help-seeking 
Table 1 Descriptive table: help-seeking knowledge score (Switzerland 2010)

\begin{tabular}{|c|c|c|c|c|}
\hline Help-seeking knowledge score & Mean & SE & $95 \% \mathrm{CI}$ & Total $n$ \\
\hline Total & 7.12 & 0.03 & $7.06-7.19$ & 2,614 \\
\hline \multicolumn{5}{|l|}{ Gender } \\
\hline Men & 7.05 & 0.05 & $6.96-7.14$ & 1,305 \\
\hline Women & 7.19 & 0.05 & $7.10-7.29$ & 1,309 \\
\hline \multicolumn{5}{|l|}{ Age (years) } \\
\hline $17-34$ & 7.16 & 0.04 & $7.07-7.24$ & 1,575 \\
\hline $35-49$ & 7.19 & 0.06 & $7.07-7.31$ & 758 \\
\hline $50+$ & 6.75 & 0.10 & $6.55-6.96$ & 281 \\
\hline \multicolumn{5}{|l|}{ Region of origin } \\
\hline Portugal & 7.61 & 0.09 & $7.44-7.78$ & 450 \\
\hline Turkey & 7.25 & 0.06 & $7.13-7.38$ & 651 \\
\hline Serbia & 7.35 & 0.08 & $7.19-7.52$ & 462 \\
\hline Kosovo & 6.51 & 0.06 & $6.40-6.63$ & 651 \\
\hline Naturalised Turkey & 7.35 & 0.11 & $7.14-7.56$ & 200 \\
\hline Naturalised Kosovo & 6.81 & 0.11 & $6.59-7.03$ & 200 \\
\hline \multicolumn{5}{|l|}{ Duration of stay (years) } \\
\hline $0-1$ & 7.09 & 0.07 & $6.95-7.24$ & 500 \\
\hline $2-4$ & 7.05 & 0.09 & $6.88-7.21$ & 342 \\
\hline $5-9$ & 7.20 & 0.09 & $7.02-7.37$ & 446 \\
\hline $10+$ & 7.13 & 0.05 & $7.04-7.22$ & 1,326 \\
\hline \multicolumn{5}{|l|}{ Education } \\
\hline Low education & 6.89 & 0.05 & $6.80-6.99$ & 1,117 \\
\hline Medium education & 7.17 & 0.05 & $7.07-7.26$ & 1,229 \\
\hline High education & 7.93 & 0.11 & $7.72-8.14$ & 259 \\
\hline
\end{tabular}

knowledge in the case of either minor or major symptoms: a higher score for recommending a consultation even for minor symptoms indicated potential overuse (five scenarios) and a higher score not recommending a consultation in case of severe symptoms indicated potential underuse (seven scenarios).

The basic distribution of the total score is displayed in means, standard errors and $95 \%$ confidence intervals (Table 1). We used three categories for age, and four for years of stay. To explore basic social and cultural variations in immigrants' knowledge about when to seek professional help, we applied a linear regression model that estimated the effects of the following explanatory variables: duration of stay in Switzerland (increment 1 year); gender; age (increment 1 year); region of origin/immigration status (Portugal, Turkey, Serbia, Kosovo; naturalised immigrants from Turkey, naturalised immigrants from Kosovo); and, education. All educational degrees acquired in Switzerland or abroad were counted and ranked from low (no or only compulsory schooling) to medium (secondary education including of vocational training) and high (tertiary education). To control for confounding, we included in the model a standard measure of self-rated health (Moriarty et al. 2003).

\section{Analysis}

Descriptive statistics showed that it was more difficult for a respondent to give correct answers to some items than to others. The proportion of correct answers across the 12 symptoms varied within the expected range. Both of these findings supported the construct validity of our items. Combined, all items showed a normal distribution. As described above, the 12 measures were applied as formative items, to assess one particular form of knowledge. The 12 responses were added to a sum total, named "helpseeking knowledge". One point was given for each correct answer. Incorrect answers, "don't know" (1.07-5.28 \%), and "no answer," (0.11-0.50\%) were counted as 0 points. The total score for help-seeking knowledge ranged from 0 to 12. Each wrong answer in the two sub-scores received one point. The responses to the five minor and seven major symptoms were added up separately. The sub-score of knowledge deficits that may be related to overuse ranged from 0 to 5; that for potential underuse ranged from 0 to 7 .

All analyses were conducted using Stata 10.1 statistical software. Oral informed consent was obtained from participants prior to data collection. No rewards were offered for participation in this study. Ethical approval for this population survey was not required, since participation in the telephone survey was voluntary and included no personal identification data.

\section{Results}

The help-seeking knowledge score (12 items) showed a normal distribution; the mean was 7.12 points $(95 \% \mathrm{CI}$ 7.06-7.19), $n=2,614$.

Table 1 shows mean scores for help-seeking knowledge, by gender, age, region of origin, duration of stay, and education. The $95 \%$ confidence intervals revealed significant differences in help-seeking knowledge for education, some differences for age, and differences for some regions of origin, but none for gender or duration of stay. Between low and medium education, the mean score increased by 0.22 points; between medium and high education it increased by 0.62 points. For the Kosovar region of origin, the mean score was 6.70 (95\% CI 6.57-6.82); for naturalised Kosovars it was 6.97 (95\% CI 6.73-7.21). Kosovar respondents differed significantly from all other groups except naturalised Turks, and achieved the lowest scores.

In a multivariable linear regression analysis (Table 2), controlling for self-rated health, we examined the 
Table 2 Help-seeking knowledge, potential over- and potential underuse (Switzerland 2010) (Multivariable Linear Regression Analysis)

\begin{tabular}{|c|c|c|c|c|c|}
\hline \multicolumn{6}{|l|}{ Help-seeking knowledge } \\
\hline$n=2,601$ & Coeff. & SE & $p$ value & $95 \% \mathrm{CI}$ & $\beta$ Coeff \\
\hline \multicolumn{6}{|l|}{ Total score } \\
\hline Duration of stay (years) & 0.01 & 0.00 & 0.130 & 0.00 to 0.01 & 0.03 \\
\hline Gender & 0.21 & 0.06 & 0.001 & 0.08 to 0.33 & 0.06 \\
\hline Age (years) & -0.01 & 0.00 & 0.001 & -0.02 to 0.00 & -0.07 \\
\hline Turkey (ref. PT) & -0.48 & 0.10 & 0.000 & -0.68 to -0.28 & -0.12 \\
\hline Serbia (ref. PT) & -0.41 & 0.11 & 0.000 & -0.62 to -0.20 & -0.09 \\
\hline Kosovo (ref. PT) & -1.20 & 0.10 & 0.000 & -1.40 to -1.00 & -0.31 \\
\hline Naturalised Turkey (ref. PT) & -0.39 & 0.14 & 0.006 & -0.66 to -0.11 & -0.06 \\
\hline Naturalised Kosovo (ref. PT) & -0.97 & 0.14 & 0.000 & -1.24 to -0.70 & -0.15 \\
\hline Self-rated health (5 cat.) & 0.09 & 0.04 & 0.032 & 0.01 to 0.17 & 0.05 \\
\hline Education (3 cat.) & 0.41 & 0.05 & 0.000 & 0.31 to 0.51 & 0.15 \\
\hline Constant & 6.61 & 0.28 & 0.000 & 6.06 to 7.16 & \\
\hline \multicolumn{6}{|c|}{ Potential overuse score (minor symptoms) } \\
\hline Duration of stay (years) & -0.02 & 0.00 & 0.000 & -0.03 to -0.02 & -0.15 \\
\hline Gender & 0.15 & 0.05 & 0.007 & 0.04 to 0.25 & 0.05 \\
\hline Age (years) & 0.02 & 0.00 & 0.000 & 0.02 to 0.03 & 0.19 \\
\hline Turkey (ref. PT) & 0.93 & 0.09 & 0.000 & 0.77 to 1.10 & 0.28 \\
\hline Serbia (ref. PT) & 0.44 & 0.09 & 0.000 & 0.26 to 0.62 & 0.12 \\
\hline Kosovo (ref. PT) & 0.95 & 0.09 & 0.000 & 0.78 to 1.12 & 0.28 \\
\hline Naturalised Turkey (ref. PT) & 1.13 & 0.12 & 0.000 & 0.89 to 1.36 & 0.21 \\
\hline Naturalised Kosovo (ref. PT) & 0.87 & 0.12 & 0.000 & 0.64 to 1.10 & 0.16 \\
\hline Self-rated health (5 cat.) & -0.13 & 0.03 & 0.000 & -0.20 to -0.06 & -0.08 \\
\hline Education (3 cat.) & -0.13 & 0.04 & 0.003 & -0.21 to -0.04 & -0.06 \\
\hline Constant & 2.06 & 0.24 & 0.000 & 1.59 to 2.52 & \\
\hline \multicolumn{6}{|c|}{ Potential underuse score (major symptoms) } \\
\hline Duration of stay (years) & 0.02 & 0.00 & 0.000 & 0.01 to 0.03 & 0.09 \\
\hline Gender & -0.35 & 0.07 & 0.000 & -0.49 to -0.22 & -0.10 \\
\hline Age (years) & -0.01 & 0.00 & 0.000 & -0.02 to -0.01 & -0.09 \\
\hline Turkey (ref. PT) & -0.45 & 0.11 & 0.000 & -0.66 to -0.24 & -0.11 \\
\hline Serbia (ref. PT) & -0.03 & 0.12 & 0.764 & -0.26 to 0.19 & -0.01 \\
\hline Kosovo (ref. PT) & 0.25 & 0.11 & 0.021 & 0.04 to 0.46 & 0.06 \\
\hline Naturalised Turkey (ref. PT) & -0.74 & 0.15 & 0.000 & -1.03 to -0.44 & -0.11 \\
\hline Naturalised Kosovo (ref. PT) & 0.10 & 0.15 & 0.505 & -0.19 to 0.39 & 0.01 \\
\hline Self-rated health (5 cat.) & 0.04 & 0.04 & 0.335 & -0.04 to 0.13 & 0.02 \\
\hline Education (3 cat.) & -0.28 & 0.05 & 0.000 & -0.38 to -0.17 & -0.10 \\
\hline Constant & 3.33 & 0.30 & 0.000 & 2.74 to 3.92 & \\
\hline
\end{tabular}

associations of duration of stay, gender, age, region of origin and education, with the outcomes help-seeking knowledge (12 items), knowledge deficits potentially related to overuse (5 items) and underuse ( 7 items). Helpseeking knowledge scores (our main outcome) were highest for Portuguese respondents; thus, we used them as reference group.

Help-seeking knowledge was significantly associated with gender, age, region of origin and education $(p<0.05$ for all variables) but not with duration of stay.
Compared to immigrants of Portuguese origin, immigrants from all other regions had significantly $(p<0.05)$ lower total scores on help-seeking knowledge. This difference was largest for Kosovar immigrants (-0.97 and -1.20 points), followed by Turkish $(-0.39$ and -0.48 points) and Serbian immigrants ( -0.41 points).

The knowledge of Kosovar and Turkish immigrants about minor symptoms (potential overuse) was particularly low when compared to that of Portuguese immigrants. However, the knowledge of Turkish immigrants about 
major symptoms (potential underuse) was better than that of the reference group $(-0.45$ points for Turkish immigrants and -0.74 points for naturalised Turkish immigrants), $(p<0.05)$. Kosovar immigrants were more likely to have knowledge deficits potentially related to underuse than were Portuguese immigrants ( 0.25 points). Naturalised Kosovar immigrants and Serbian immigrants did not differ significantly from the reference group.

As expected, higher educational degree was positively associated with help-seeking knowledge ( 0.41 points per category). Accordingly, higher educational degree was negatively associated with knowledge deficits linked to potential overuse $(-0.13$ points) and potential underuse $(-0.28$ points $)$. Female gender moderately increased the help-seeking knowledge score ( 0.21 points on average). On the other hand, women had more knowledge deficits on minor symptoms (potential overuse) than did men $(0.15$ points), while men had knowledge deficits on major symptoms (potential underuse).

The effect of age was small, but because the increment by which age increased was 1 year, a substantial increase in age did lead to a noticeable decrease in help-seeking knowledge (e.g., -0.30 points for an additional 30 years). The additional 30 years increased knowledge deficits on minor symptoms (potential overuse) by 0.60 points, and decreased the knowledge score on major symptoms (potential underuse) by 0.30 points. Longer duration of stay in the host country (Switzerland) was not significantly associated with help-seeking knowledge, but adding 20 years to length of stay decreased the knowledge score for potential overuse by 0.40 points, and increased it by the same amount for potential underuse.

\section{Discussion}

Public Health research on health literacy is rapidly increasing, and significant advances have been made in conceptual work, measurement development and monitoring. To date, we see an increasing number of differentiated approaches to health literacy applied in different fields, such as in medical care settings (Abel 2008; Nutbeam 2008; Peerson and Saunders 2009), or health literacy in the private realm (Abel et al. 2014; Dermota et al. 2013). The present study addressed one important component in applied health literacy, namely, people's knowledge on when to seek professional help.

We applied a set of 12 questions that assessed knowledge on when to seek professional help for common health problems (physical and psychological symptoms). We assume the scenarios we chose are not limited to Switzerland, but also apply in regions with less-developed or less well-equipped health care systems, such as those from which our study participants originated. We studied the distribution of functional health literacy across four migrant groups in Switzerland. In a multivariable linear regression analysis that controlled for self-rated health, we found that region of origin, higher education, female gender and younger age were significantly and independently associated with better help-seeking knowledge scores.

Our ability to discuss our findings in the context of other studies was limited because research on the link between region of origin, ethnic background of migrants and health literacy is still scarce. This lack became even more evident when we looked for empirical studies that specifically focused on knowing when to seek professional help for common health problems.

When we searched for differences by region of origin, our analyses of the total knowledge score showed that Portuguese respondents had the highest scores for knowing when to seek help, while Kosovar immigrants had the lowest. We used our sub-scores to assess two types of knowledge deficits and found that Turkish immigrants tended to recommend professional help even for minor health problems (potential overuse). Kosovar immigrants more generally misjudged symptoms, which suggest they might both overuse and underuse health services. We are not aware of studies that could help us interpret the findings from the Kosovar sample.

Our Turkish sample had a relatively high score for seeking professional help for only minor symptoms, and this aligns with a Dutch study that found Turkish immigrants use health services more often than the native population does, even after controlling for self-rated health (Uiters et al. 2006). In contrast, another Dutch study (Lamkaddem et al. 2011) discovered a contrary effect in Turkish (and Moroccan) immigrants that showed potential underuse, related to perceived discrimination. These two studies are examples of conflicting results about healthcare utilization rates for Turkish and other migrant populations. Over- or underuse seems to depend on the type of care, organisation, and other aspects of health care services. Since there are so few studies on health literacy in immigration populations, we could observe but not explain the variations we found across the ethnic groups. Variation in help-seeking knowledge warrants further research. Our results on differences in knowledge, potentially relevant to over- and underuse, could provide useful starting points for future qualitative and quantitative studies.

In line with previous studies, we found individuals with higher education were more likely to know when to seek professional help (Hetherington and Hopkins 1969; Stoeckle et al. 1963; Turner and Nido 1988). Other studies found that individuals with more formal education tended to be more health literate (Fransen et al. 2011) and made better and more appropriate use of health services 
(Berkman et al. 2011). Our findings on the effects of education on knowledge about when to seek professional help for common health problems fit well with evidence that links education to health literacy, and health literacy to use of healthcare. Moreover, the effect of education was independent of cultural background. Since a large proportion of respondents acquired their educational degree in their countries of origin, this indicates that higher education, achieved before or after migration, has a positive effect on the form of health literacy that we examined in this study.

We found an association between age and help-seeking knowledge: as the former increased, the latter decreased. Specifically, as immigrants aged, their knowledge scores were more likely to indicate overuse, and less likely to indicate underuse. In older age, recommending professional consultation even for minor symptoms may be a reasonable approach. With increasing age, minor symptoms may be perceived as signals or precursors of severe health problems. This interpretation fits with the higher knowledge scores we found among older immigrants of recommending professional help for major symptoms.

Previous studies have found women generally report more symptoms and rate their own health worse than men (Andersen et al. 1968; Darviri et al. 2012). Women also use health services more often than men do (Mansfield and Addis 2003). We found immigrant women, overall, more likely than men to know when to seek help for common health problems. Women in our sample were more likely to answer in favour of seeing a professional, even for minor symptoms, and less likely to fail to recommend a consultation in case of major symptoms. The literature on migrant health behaviours neither directly supports nor contradicts these findings on gender differences.

We thought that the amount of time immigrants spent in their host country could have affected their help-seeking knowledge, so we searched the data for evidence to support this assumption. We found that longer duration of stay was significantly associated with knowledge potentially related to overuse and underuse. Acculturation, or a learning effect, could explain a decline in potential overuse as duration of stay lengthened. It was more difficult to explain the tendency to underuse that we saw in the answers of respondents with longer-term residence. It is possible that the amount of discrimination immigrants perceive increases over time. A Dutch study that analysed non-attendance to general practitioners found evidence of this effect (Lamkaddem et al. 2011). It is also possible that developing stronger interconnectedness and more efficient social networks in the host country opens up new channels of information and augments lay referral systems. These could reassure migrants and encourage them to dispense with physician consultations-even beyond the point of making a medically correct decision. More research is needed to test possible explanations and evaluate effects of length of stay on help-seeking knowledge and healthcare utilization.

There were several limitations in the current study that need to be taken into account. The data were cross-sectional, which allowed associations to be analysed, but not causal inferences. We established content validity of our symptom items through expert ratings, but we had no measures available to test for criterion and predictive validity of our sum scores. No native Swiss sample was available with this form of data, limiting our findings on the distribution and the determinants to migrant populations only. We studied only subjective assessments, in the form of advice to seek professional help. While subjective assessments are an important element in lay referral systems (Jones et al. 1981), they should not be interpreted as measures of actual behaviour. We described basic variations in social factors only across the different migrant groups. Future studies should also look at the effect of social determinants (education etc.) within each migrant group.

\section{Conclusion}

Our study was based on a large and diverse immigrant sample from four European regions of origin, providing us with a statistically sound basis for producing the first population-based empirical evidence that social and cultural background and help-seeking knowledge are associated in Swiss immigrants. By applying two subscores, we were able to detect varying distribution patterns of knowledge relevant to potential overuse and underuse.

Our results provide population-based empirical support for Jordan's et al. concept of health literacy, which considers individuals' knowledge on when to seek professional help to be a key element in successfully handling health problems. We identified ethnic background and other social determinants as relevant co-factors for the knowledge needed to successfully deal with health issues in everyday life. As such our findings may help to advance current theoretical and conceptual approaches in health literacy research. Our results contribute to the empirical knowledge base, but more studies are needed to improve our understanding of the distribution of health literacy among migrant populations.

\section{References}

Abel T (2008) Measuring health literacy: moving towards a healthpromotion perspective. Int J Public Health 53:169-170

Abel T, Hofmann K, Ackermann S, Bucher S, Sakarya S (2014) Health literacy among young adults: a short survey tool for 
public health and health promotion research. Health Promot Int. doi:10.1093/heapro/dat096 (First published online: January 30, 2014)

Andersen R, Anderson O, Smedby B (1968) Perception of and response to symptoms of illness in Sweden and the United States. Med Care VI(1):18-30

Berkman ND, Sheridan SL, Donahue KE, Halpern DJ, Crotty K (2011) Low health literacy and health outcomes: an updated systematic review. Ann Intern Med 155(2):97-107

Cha ES et al (2012) Understanding cultural issues in diabetes selfmanagement behaviors of Korean immigrants. Diabetes Educ 38(6):835-844

Cockerham WC, Lueschen G, Kunz G, Spaeth JL (1986) Social stratification and self-management of health. J Health Social Behav 27(1):1-14

Cooper M, Harding S, Mullen K, O'Donnell C (2012) 'A chronic disease is a disease which keeps coming back...it is like the flu': chronic disease risk perception and explanatory models among French- and Swahili-speaking African migrants. Ethn Health 17(6):597-613

Darviri C, Fouka G, Gnardellis C, Artemiadis AK, Tigani X, Alexopoulos EC (2012) Determinants of self-rated health in a representative sample of a rural population: a cross-sectional study in Greece. Int J Environ Res Public Health 9(3):943-954

Dermota P, Wang J, Dey M, Gmel G, Studer J, Mohler-Kuo M (2013) Health literacy and substance use in young Swiss men. Int $\mathbf{J}$ Public Health 58:939-948

Dunn-Navarra A-M, Stockwell MS, Meyer D, Larson E (2012) Parental health literacy, knowledge and beliefs regarding upper respiratory infections (URI) in an urban Latino immigrant population. J Urban Health 89(5):848-860

Edwards JR, Bagozzi RP (2000) On the nature and direction of relationships between constructs and measures. Psychol Methods 5(2):155-174

Fransen MP, Van Schaik TM, Twickler TB, Essink-Bot ML (2011) Applicability of internationally available health literacy measures in the Netherlands. J Health Commun 16(Suppl 3):134-149

Guggisberg $J$ et al (2011) Gesundheitsmonitoring der Migrationsbevölkerung in der Schweiz (Health monitoring of the migrant population in Switzerland). http://www.bag.admin.ch/themen/ gesundheitspolitik/07685/12533/12535/index.html?lang=de

Hetherington RW, Hopkins CE (1969) Symptom sensitivity: its social and cultural correlates. Health Serv Res 4(1):63-75

Jones RA, Wiese HJ, Moore RW, Haley JV (1981) On the perceived meaning of symptoms. Med Care 19(7):710-717

Jordan JE, Buchbinder R, Osborne RH (2010) Conceptualising health literacy from the patient perspective. Patient Educ Couns 79(1):36-42

Kickbusch IS (2001) Health literacy: addressing the health and education divide. Health Promot Int 16(3):289-297

Lamkaddem M, Essink-Bot ML, Devillé W, Foets M, Stronks K (2011) Perceived discrimination outside health care settings and health care utilization of Turkish and Moroccan GP patients in the Netherlands. Eur J Public Health 22(4):473-478

Mansfield AK, Addis ME (2003) "Why won't he go to the doctor?": the psychology of men's help seeking. Int J Men's Health 2(2):93-109
Moriarty D, Zack M, Kobau R (2003) The centers for disease control and prevention's healthy days measures-population tracking of perceived physical and mental health over time. Health Qual Life Outcomes 1(1):37

Ng E, Pottie K, Spitzer D (2011) Official language proficiency and self-reported health among immigrants to Canada. Health Rep 22(4):15-23

Nutbeam D (2000) Health literacy as a public health goal: a challenge for contemporary health education and communication strategies into the 21st century. Health Promot Int 15(3):259-267

Nutbeam D (2008) The evolving concept of health literacy. Soc Sci Med 67(12):2072-2078

Omariba DW, Ng E (2011) Immigration, generation and self-rated health in Canada: on the role of health literacy. Can J Public Health 102(4):281-285

Peerson A, Saunders M (2009) Health literacy revisited: what do we mean and why does it matter? Health Promot Int 24(3):285-296

Poureslami I, Rootman I, Doyle-Waters MM, Nimmon L, Fitzgerald JM (2011) Health literacy, language, and ethnicity-related factors in newcomer asthma patients to Canada: a qualitative study. J Immigr Minor Health 13(2):315-322

Ratzan SC, Parker RM (2000) Introduction. In: Selden CR, Zorn M, Ratzan SC, Parker RM (eds) National library of medicine current bibliographies in medicine: health literacy. National Institutes of Health, US Department of Health and Human Services, Bethesda

Schulz PJ, Nakamoto K (2005) Emerging themes in health literacy. Stud Commun Sci 5:1-10

Shaw SJ, Huebner C, Armin J, Orzech K, Vivian J (2009) The role of culture in health literacy and chronic disease screening and management. J Immigr Minor Health 11:460-467

Smith PC, Brice JH, Lee J (2012) The relationship between functional health literacy and adherence to emergency department discharge instructions among Spanish-speaking patients. J Natl Med Assoc 104(11-12):521-527

Stoeckle JD, Zola IK, Davidson GE (1963) On going to see the doctor, the contributions of the patient to the decision to seek medical aid. A selective review. J Chronic Dis 16:975-989

Tieu Y, Konnert C, Wang J (2010) Depression literacy among older Chinese immigrants in Canada: a comparison with a populationbased survey. Int Psychogeriatr 22(8):1318-1326

Todd L, Hoffman-Goetz L (2011) Predicting health literacy among English-as-a-second-language older Chinese immigrant women to Canada: comprehension of colon cancer prevention information. J Cancer Educ 26(2):326-332

M.I.S. Trend SA (2011) Gesundheitsbefragung bei Personen mit Migrationshintergrund in der Schweiz (Health survey among people with migration background in Switzerland). Institut für Wirtschafts- und Sozialforschung

Turner BJ, Nido RM (1988) Urgency in seeking medical care for specific symptoms: perceptions of physicians and patients. J Gen Intern Med 3(3):245-249

Uiters E, Deville WL, Foets M, Groenewegen PP (2006) Use of health care services by ethnic minorities in The Netherlands: do patterns differ? Eur J Public Health 16(4):388-393 